\title{
Lusioersily
}

\section{Electrocardiographic Waveforms Fitness Check Device Technique for Sudden Cardiac Death Risk Screening}

Escalona, OJ., \& Mendoza, M. (2016). Electrocardiographic Waveforms Fitness Check Device Technique for Sudden Cardiac Death Risk Screening. In Unknown Host Publication (pp. 3453-3456). IEEE Xplore. https://doi.org/10.1109/EMBC.2016.7591471

Link to publication record in Ulster University Research Portal

\section{Published in:}

Unknown Host Publication

Publication Status:

Published online: 30/08/2016

DOI:

10.1109/EMBC.2016.7591471

\section{Document Version}

Publisher's PDF, also known as Version of record

\section{General rights}

Copyright for the publications made accessible via Ulster University's Research Portal is retained by the author(s) and / or other copyright owners and it is a condition of accessing these publications that users recognise and abide by the legal requirements associated with these rights.

\section{Take down policy}

The Research Portal is Ulster University's institutional repository that provides access to Ulster's research outputs. Every effort has been made to ensure that content in the Research Portal does not infringe any person's rights, or applicable UK laws. If you discover content in the Research Portal that you believe breaches copyright or violates any law, please contact pure-support@ulster.ac.uk. 


\title{
Electrocardiographic Waveforms Fitness Check Device Technique for Sudden Cardiac Death Risk Screening
}

\author{
O.J. Escalona, Member, IEEE, and M. Mendoza
}

\begin{abstract}
A novel cardiac health device technique development for reliable, non-invasive and cost-effective heart screening in preventive cardiovascular healthcare is presented. In particular, identification of apparently healthy individuals involved in sports activities (particularly in the young, age $<35$ years) who may be at-risk of sudden-cardiac-death (SCD) is mainly focused here. Nevertheless, the same device technique may be prospectively extended for detecting cardiovascular abnormalities in children and adolescents with type1-diabetes, and also in detecting patients with Brugada syndrome. The device system has been aimed to provide a single figure diagnostic output, thus, not requiring highly-skilled medical personnel. The principles of the required ECG-waveform analysis algorithm have been reported in previous clinical studies. A prototype system platform design that will enable low-cost, portability and key user-friendly characteristics was implemented and in-vitro tested. Real-time firmware integrity and cardiac fitness detection algorithm performed reliably with an in-vitro positive SCD ECG-waveform modelling technique.
\end{abstract}

\section{INTRODUCTION}

Sudden cardiac death (SCD) is a major health problem in Europe. While there are difficulties in estimating the exact incidence, in the Maastricht study that showed that during the 1990s in the Netherlands, $45 \%$ of SCD sufferers had no known history of cardiac disease and $40 \%$ had low or medium risk profiles following a heart attack [1]. It would be a significant advance if a method was devised capable of rapidly identifying a greater proportion of at risk individuals. An innovative approach could be by embedding within the related device technology the expertise required for the novel method. In this way, clinician training necessary for handling and interpreting new health devices using advanced concepts, can be minimised or not required at all. Thus benefits offered by improved, but sophisticated screening techniques are facilitated more quickly and efficiently into medical practice, leading to significant reduction on the frequency of SCD $[2,3]$. Therefore, we aim to provide a medical device technology that is user friendly, portable, and cost-effective for cardiac screening using non-invasive ECG complex waveform analysis to achieve the required reliability of the cardiac risk screening procedure. The proposed ECG waveform fitness check (ECGWFC) device, is targeted to be operator independent. The device will just provide a single measurement figure (a dimensionless number) result output per person being checked. Therefore, the number of people being heart screened can be increased

O. J. Escalona (corresponding author) is with the Engineering Research Institute and the NIBEC at the Ulster University, Newtownabbey, BT37 0QB, UK; Tel: +44 2890366151 (e-mail: oj.escalona@ulster.ac.uk).

M. Mendoza, is with Universidad Simon Bolivar, Baruta, Caracas, Venezuela (e-mail: marianela.min@gmail.com). only by increasing the number of ECGWFC units and the number of unspecialised clinicians, such as nurses or paramedics, who could be easily trained for operating the ECGWFC device and placing the required ECG electrodes (only 7 electrodes). Therefore, increasing the feasibility of the screening programme and reducing its operational costs.

\section{METHODS}

The key medical technology involved in the heart fitness check device comprises a technique of analysis of ventricular late potentials (VLP) measurements in a highresolution electrocardiographic recording using a unique ECG signal averaging (SAECG) process, named SFP (single fiducial point) [4]. SAECG improves the signal-to-noise ratio of a surface ECG, permitting the identification of VLP, which are low-amplitude (microvolt level) signals at the end of the ventricular activity [3]. A particular especial algorithm for VLP analysis quantifies a parameter related to the complexity of VLP waveforms in the $3 \mathrm{D}$ voltage space as the indicator for risk assessment $\left(\delta_{\mathrm{LP}}\right)$ [5]. This is done by computing the fractal dimension of the 3D VLP curve drawn in a high definition voltage scale (in microvolts); the voltage curve being measured on three orthogonal (X,Y and Z) ECG signal components [6]. Several clinical studies have confirmed that a fractal dimension above 1.3, can be selected as the threshold value indicating risk of SCD in the subject being checked for heart fitness [5]. The reported standard deviation $(\sigma)$ of $\delta_{\mathrm{LP}}$ in the at-risk groups being about 0.08 , and in the non at-risk groups being about 0.06, with difference between means ranging about twice those values $\left(\mu_{1}-\mu_{2} \approx 2 \sigma\right)$. The fractal dimension quantification parameter $\delta_{\text {LP }}$ may be provided as a numerical display, or just as a simple SCD risk warning lamp (green/red) on the device, depending on whether the value of $\delta_{\mathrm{LP}}$ is below/above a threshold value (e.g., a value of 1.3)[5].

\section{A. ECGWFC System Implementation}

A real-time ECGWFC device system was implemented for VLP isolation and $\delta_{\mathrm{LP}}$ analysis. It is integrated by two main components: the hardware and the software subsystems. The hardware subsystem includes rechargeable battery power supply, 3-channel low-noise ECG amplification, analogue signal conditioning, analogue to digital converters (ADC), embedded ECG processing optoisolation and interface chipset, and the portable PC (laptop) subsystem. The orthogonal bipolar XYZ lead system was adopted for recording the ECG signals [2].

As described for previous in-house high-resolution ECG acquisition system [6], the overall gain was set to 2000 . Analogue signal conditioning was implemented by $1^{\text {st }}$ order, 
$3 \mathrm{~Hz}$ high-pass filtering at the front-end instrumentation amplifier, and low-pass filtering by an antialiasing $5^{\text {th }}$ order filter at $360 \mathrm{~Hz}$. Thus, the 3-channel front-end analogue ECG acquisition stage had a bandwidth from 3 up to $360 \mathrm{~Hz}$. Digitisation was carried out at $2 \mathrm{kHz}$ sampling frequency and at 16-bit resolution (ADC).

A micro-controller based firmware implemented the signal processing that enabled a highly accurate real-time alignment reference in the QRS complex for signal averaging (SAECG). The Single-Fiducial-Point (SFP) alignment technique algorithm [4] was coded to carry out this real-time task. Thus, the $\mathrm{X}, \mathrm{Y}$ and $\mathrm{Z}$ ECG channels data plus a QRS alignment reference bit data were output to the portable PC/laptop via the USB communication port. A LabView application code was implemented in the PC subsystem to provide the operator interface console, and to perform some required high level computational processes. SAECG and VLP Isolation

The micro-controller embedded SFP alignment technique facilitated the real-time computation of the three SAECG frames; one for each of the orthogonal leads $(X, Y, Z)$. In this process, the reference lead and the number of averaged beats were manually selected by the operator. Then, conventional digital high-pass filtering at $40 \mathrm{~Hz}$ was implemented to generate the standard filtered SAECG frames for conventional VLP analysis (bi-directional, $4^{\text {th }}$ order Butterworth high-pass digital filtering) on each lead: $F X, F Y$ and $F Z[7]$.

VLP isolation was implemented as in previous works [6]. For this, first the $\mathrm{X}_{\mathrm{VLP}}, \mathrm{Y}_{\mathrm{VLP}}$ and $\mathrm{Z}_{\mathrm{VLP}}$ vector components are defined based on start and end timing marks obtained from the combined vector magnitude array $\boldsymbol{A}$ from the above filtered SAECG frames of each orthogonal lead, as defined in equation (1).

$$
\boldsymbol{A}=\sqrt{F X^{2}+F Y^{2}+F Z^{2}}
$$

In the computed vector magnitude array $\boldsymbol{A}$, the end timing mark ( $\left.\mathrm{t}_{\text {end }}\right)$ of VLP activity was located at the moment when the of values in $\boldsymbol{A}$ becomes three times the background noise level in the ST segment region of the SAECG; and the start timing mark ( $\left.\mathrm{t}_{\text {start }}\right)$ was located when the values in $\boldsymbol{A}$ become greater than $40 \mu \mathrm{V}$, in reverse time sequence move from the $\mathrm{t}_{\text {end }}$ mark. In this process, values of $\boldsymbol{A}$ are averaged in a 10 ms window, in $5 \mathrm{~ms}$ steps towards the QRS complex region.

Then, the segments of each orthogonal filtered SAECG ( $F X, F Y$ and $F Z$ ), delimited by the two time marks ( $\left.\mathrm{t}_{\mathrm{end}}, \mathrm{t}_{\text {start }}\right)$, define the $X_{V L P}, Y_{V L P}$ and $Z_{V L P}$ vectors respectively, that is, the isolated VLP activity in each orthogonal lead.

\section{B. Computation of the $\delta_{L P}$ parameter}

Following the VLP demarcation process and the definition of vectors $\mathrm{X}_{\mathrm{VLP}}, \mathrm{Y}_{\mathrm{VLP}}$ and $\mathrm{Z}_{\mathrm{VLP}}$, numerically scaled in microvolts, two important quantities are calculated in the 3D attractor constructed from these orthogonal filtered VLP components in the microvoltage space, in order to calculate the $\delta_{\text {LP }}$ parameter of VLP activity, which is used to detect patients that are at risk of SCD. In our proposed method, we estimated the fractal dimension of the above attractor, according to Mandelbrot estimation formula:

$$
\delta_{L P} \approx \frac{\log (C)}{\log (\boldsymbol{\Phi})}
$$

In this formula, $C$ would be the total length of the attractor's 3D trajectory, and $\boldsymbol{\Phi}$ would be the spherical maximum possible diameter of the VLP attractor. Parameters $C$ and $\boldsymbol{\Phi}$ are therefore measured in $\mu \mathrm{V}$ units for properly computing the $\delta_{\text {LP }}$ parameter. The value of $C$ was calculated using the following summatory expression:

$C=\sum_{i=2}^{i=K} \sqrt{(F X(i)-F X(i-1))^{2}+(F Y(i)-F Y(i-1))^{2}+(F Z(i)-F Z(i-1))^{2}}$

In where $K$ is the number of sampling periods in the delimited VLP attractor time interval given by: $T=\left(\mathrm{t}_{\mathrm{end}}-\right.$

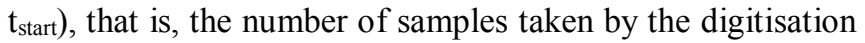
process in the interval $T$. The determination of parameter $\boldsymbol{\Phi}$ is computationally more intensive, but not complicated. For every pair of points in the 3D VLP attractor, the respective distance between them is computed using equation (4), and each calculated value used to populate the elements of the distance matrix $\Phi_{m n}$ defined in (5); then the maximum element value in $\Phi_{m n}$ yields the value of parameter $\boldsymbol{\Phi}$.

$$
\begin{gathered}
\Phi_{m n}=\sqrt{(F X(m)-F X(n))^{2}+(F Y(m)-F Y(n))^{2}+(F Z(m)-F Z(n))^{2}} \\
\boldsymbol{\Phi}=\operatorname{Max}\left(\Phi_{m n}\right)
\end{gathered}
$$

Because the distance matrix $\boldsymbol{\Phi}_{\boldsymbol{m} n}$ is symmetric, due to the square functions in the eq. (4), and zero diagonal elements resulting when $m=n$, only half of $\boldsymbol{\Phi}_{\boldsymbol{m} n}$ elements need to be calculated using eq. (4) to find the value of parameter $\boldsymbol{\Phi}$. Finally, as indicated above, our previous studies have suggested that a value above 1.3 of parameter $\delta_{\text {LP }}$ would be a reliable indication of a positive case of SCD risk.

\section{ECGWFC System Performance Evaluation}

A preliminary bench based assessment of the ECGWFC device system was implemented. For this, a $60 \mathrm{~ms}$ width QRS pulse model generated at a rate of $80 \mathrm{bpm}$, and of about $1 \mathrm{mV}$ amplitude, was used as a modelled ECG signal, input into channel $\mathrm{X}$, as the averaging reference lead. Because the WCGWFC device ECG amplifiers operate with a band-pass filtering from 3 to $360 \mathrm{~Hz}$, our modelled QRS signal is transformed into the biphasic pulse shown in Figure 1.

\section{1 Noise Immunity}

Spectral degradation upon the SAECG due to noise interference effect on the SFP (Single-Fiducial-Point) alignment technique was assessed by means of the modelled ECG and deducing the standard deviation (SD) of alignment error (jitter), as a result of added noise into the modelled ECG signal input into the $\mathrm{X}$ channel of our ECGWFC device under bench test. Thus, noise immunity of the adopted 


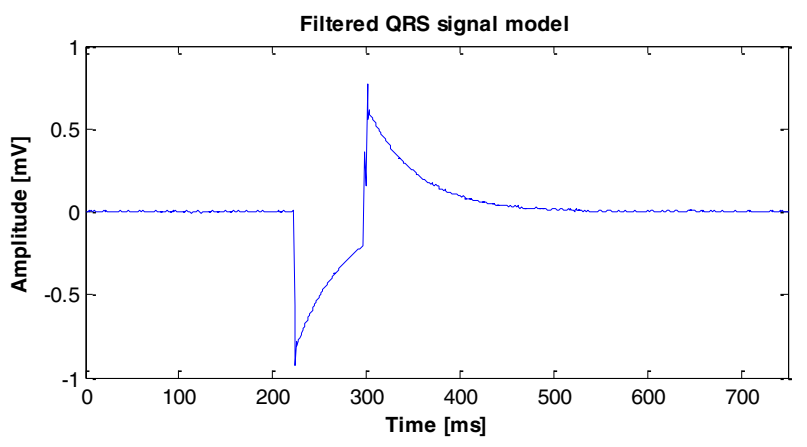

Figure 1. Synthetic QRS signal model.

SAECG processing technique, under increasing noisy conditions was assessed under controlled adverse situations that results into spectral degradation of the SAECG process. For this, a noiseless (uncontaminated) modelled ECG signal was simultaneously fed into the $\mathrm{Y}$ or $\mathrm{Z}$ channels, while the controlled noisy ECG model signal was fed into the selected reference channel $\mathrm{X}$. The types of simulated added noise were EMG and $50 \mathrm{~Hz}$ mains interference. The embedded SFP algorithm attempts to generate the beat alignment timing mark, but with a corresponding noise effect error. The latter error in every beat is measured with respect to any of the noiseless input channels ( $\mathrm{Y}$ or $\mathrm{Z}$ ), carrying the information of the true moment of the QRS pulse position. Thus, by means of a simple noise immunity assessment algorithm, vectors of beat alignment errors (jitter) were generated for each controlled type and rms noise level condition of test. The assessment algorithm also computed the SD value of each jitter vector case. For the ECGWFC device operating sampling frequency of $2 \mathrm{kHz}$, a jitter vector SD value below $0.5 \mathrm{~ms}$, was considered as good noise immunity, and with negligible spectral degradation effects in the SAECG process.

\section{D.2 ECG Denoising Performance}

Assessment of the ECGWFC device required ECG denoising functionality was implemented in a controlled bench testing method, using the two types of simulated noises used for the noise immunity assessment: simulated EMG (band limited Gaussian noise) and $50 \mathrm{~Hz}$ interference (sinusoidal). In a similar manner, the modelled ECG signal was corrupted with seven stepped up noise levels and fed into the selected reference channel $\mathrm{X}$ of the ECGWFC device. Then, ECG denoising performance at each level and type of added noise was assessed by measuring progressive noise reduction by the SAECG process at 7 stages of increasing number of averaged beats, from 1 to 400 . The expected performance profile for Gaussian type noise (simulated EMG), is a noise reduction that is inversely proportional to the square root of the number of averaged beats.

\section{D.3 In-vitro $\delta_{L P}$ Measurement Performance}

The ECGWFC device was tested in several in-house healthy volunteers $(\mathrm{N}=5)$. Thus, VLP activity in these small sample healthy cases was measured by the $\delta_{\mathrm{LP}}$ parameter output by the ECGWFC device, in order to confirm the expected values of $\delta_{\mathrm{LP}}$ characteristic in healthy subject cases. To emulate high VLP activity conditions in-vitro, ECG synchronised 3D model VLP signal was analogically generated using a filters bank network. Therefore, the objective of the proposed $\delta_{\mathrm{LP}}$ measurement assessment method was to provide in-vitro SCD positive ECG waveforms presenting rather complex 3D, VLP signal components at the input of the ECGWFC device under test. The 3D model VLP signal may also consist of three different bipolar wavelet pulses or resonant waveforms at natural frequencies ranging from $40 \mathrm{~Hz}$ to $120 \mathrm{~Hz}$. A range of $\delta_{\mathrm{LP}}$ values, at abnormal levels between 1.32 and 1.38 (see Figure 2 ), was obtained by varying the amplitude of the ECG synchronised input pulse generation between $3 \mathrm{~V}$ and $18 \mathrm{~V}$.

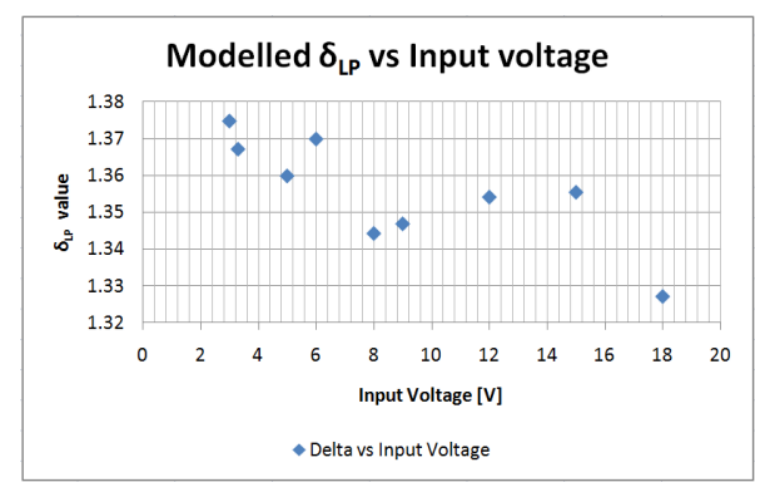

Figure 2. Modelled $\delta_{\mathrm{LP}}$ values obtained as the ECG synchronised input pulse amplitude is adjusted between $3 \mathrm{~V}$ and $18 \mathrm{~V}$.

\section{RESULTS}

\section{A. SAECG Spectral Degradation vs Noise}

Figure 3 summarises de results obtained in the noise immunity bench assessment of the ECGWFC device, for both types of noise: Gaussian and $50 \mathrm{~Hz}$. The SD value of each jitter (beat alignment error) vector case resulting from the SAECH process was measured by the above mentioned noise assessment algorithm. EMG noise was Gaussian at 300 $\mathrm{Hz}$ bandwidth. With Gaussian type noise, values of theoretically deduced spectral degradation may be estimated from alignment jitter SD values by the following

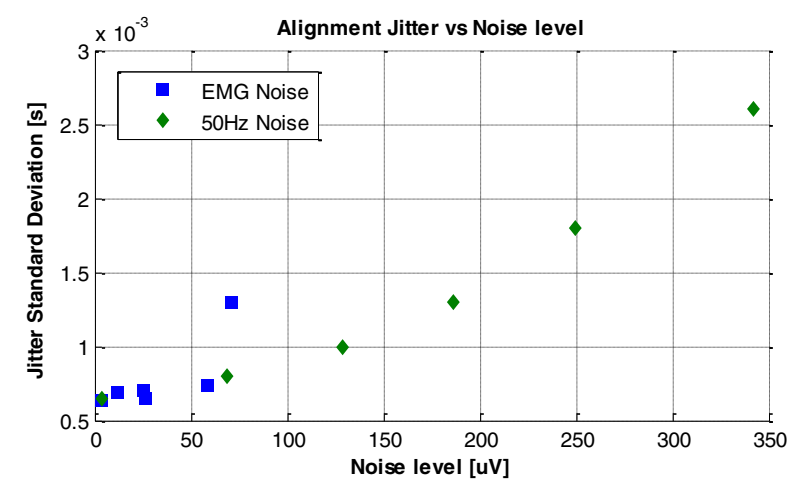

Figure 3. Beat alignment jitter resulting from added $\mathrm{EMG}$ and $50 \mathrm{~Hz}$ noise levels to a clean signal. 
relation $\mathrm{BW}=(0.13 /(\mathrm{SD}$ jitter $))$ [4]. For example, a Gaussian noise level of $70.1 \mu \mathrm{V}$ (rms) can yield a $100 \mathrm{~Hz}$ BW limitation at the output of the SAECG process. Also, from Figure 3, it can be observed that a high $50 \mathrm{~Hz}$ noise level case of $340 \mu \mathrm{V}(\mathrm{rms})$, can yield a jitter SD of $2.6 \mathrm{~ms}$. A relatively high value compared to the $0.5 \mathrm{~ms}$ reference level explained above.

With EMG noise type, linear denoising trends of the square root of number of averaged beats $(\sqrt{\mathrm{N}})$, between final noise and initial noise levels, eq. (6), was confirmed for the implemented SAECG process in the ECGWFC device.

$$
(\text { Initial noise level) }=\sqrt{N} \text { (Final noise level) }
$$

\section{B. $\delta_{L P}$ Parameter Measurement Algorithm: In-vitro Test}

To assess the functionality of the $\delta_{\mathrm{LP}}$ parameter measuring algorithm implemented in the ECGWFC device, in-vitro control measurements were carried out in five in house healthy subjects within the research team (procedures approved by the Institutional Ethical Filter). The measured values of the $\delta_{\mathrm{LP}}$ parameter in these healthy subjects (i.e., SCD -ve) were around 1.2 (mean value), and the standard deviation was of \pm 0.063 . Thus, the values of $\delta_{\mathrm{LP}}$ were all under 1.3; as expected for healthy subjects [4]. The conventional SAECG vector magnitude frame presentations were also constructed, and one of these is shown in Fig. 4(a).

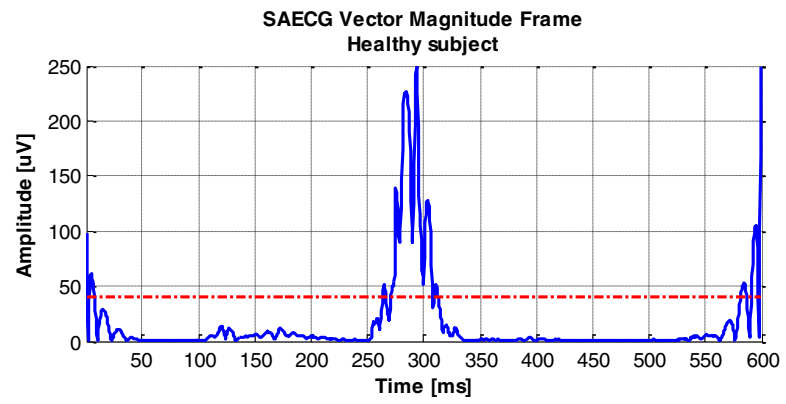

(a)

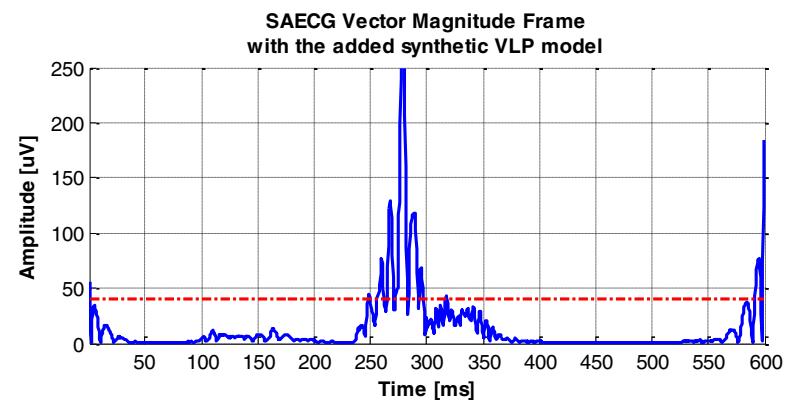

(b)

Figure 4. Filtered SAECG vector magnitude frames obtained from a healthy subject case: (a) baseline SAECG recording, (b) SAECG recording with added modelled VLP signal.

Then, after simultaneously adding the ECG synchronised VLP signal model, while the ECGWFC device recorded the body surface ECG signal again in the same healthy volunteer in Figure 4(a), the SAECG vector magnitude frame shown in Figure 4(b) was obtained. As the synthetic VLP model was of considerable complexity (to emulate SCD+ VLP activity), the measured value of the $\delta_{\text {LP }}$ parameter resulted to be 1.327 . Thus, the value of $\delta_{\mathrm{LP}}$ in this healthy subject increased from 1.198 to 1.327 after the in-vitro implementation of a SCD+ ECG-waveform modelling technique, for testing the ECGWFC device measuring algorithm.

\section{DISCUSSION AND CONCLUSIONS}

The expertise dependency nature of conventional heart screening techniques, constitutes a major hindrance to their effective and ample adoption by clinicians [8]. The number of people being screened would be limited by the number of available specialised clinical staff in the screening programme. A cardiac screening technology that is accurate, portable and cost effective is important in providing a significant contribution to solving the problem. Here, we have implemented and in-vitro tested a solution technique, in which the required advanced expertise component is embedded within the system device. With this approach, the required clinician training is minimised, aiming to facilitate the benefits offered by solution technique into the clinical practice and significantly reducing the incidence of SCD in the population. Thus, an advancement for a novel medical technology that is user friendly, portable, and cost-effective for cardiac screening using non-invasive complex waveform analysis of the electrocardiogram, has been achieved. Such a novel device will require minimal or no specialised clinician training because of its embedded or integrated processing expertise, and also its simple patient grading output figure that is easy to interpret, as it could be a simple outcome lamp indicator. Thus, the flow of heart checked people in a screening day would depend mainly on the number of available ECGWFC units and the number of minimally trained healthcare personnel.

\section{REFERENCES}

[1] J.J. Goldberger, et al. "Cardiology Committee on Electrocardiography and Arrhythmias and Council on Scientific Statement from the AHA Council on Clinical Techniques for Identifying Patients at Risk for Sudden Cardiac Death". Circulation, v. 118, 2008, pp.1497-1518.

[2] D. Corrado, C. Schmied, C. Basso, M. Borjesson, et al. "Risk of sports: do we need a pre-participation screening for competitive and leisure athletes?". Eur Heart J, Vol. 32 (8), 2011, pp. 934-44.

[3] J. K. Jongman, A. Zaidi, M. Muggenthaler, S. Sharma. "Relationship between echocardiographic right-ventricular dimensions and signalaveraged electrocardiogram abnormalities in endurance athletes," Europace, Vol. 17, 2015, pp. 1441-1448.

[4] O. Escalona, R. Mitchell, D. Balderson and D. Harron. "A fast and reliable QRS alignment technique for high-frequency analysis of the signal-averaged ECG", Medical and Biological Engineering and Computing, Vol. 31, 1993, pp. S137-S146.

[5] Mitchell RH, Escalona OJ. "Discriminating at-risk post-MI patients by fractal dimension analysis of the late potential attractor". Proceedings of the IEEE EMBS; 20(8): 1573-1575, 1998.

[6] O. Escalona, M Mendoza, et al. "Real-time system for high-resolution ECG diagnosis based on 3D late potentials fractal dimension estimation". Computing in Cardiology, v. 38, 2011, pp. 789-792.

[7] M. Mizobuchi, Y. Enjoji, S. Nakamura, et al. "Ventricular late potential in patients with apparently normal electrocardiogram; predictor of Brugada syndrome". PACE; Vol. 33 (3), 2010, pp. 266-273.

[8] B. J. Maron, E. J. Rowin, S.A. Casey, T.S. Haas, R. H. Chan, J. E. Udelson, et al., "Risk stratification and outcome of patients with hypertrophic cardiomyopathy $>=60$ years of age." Circulation, Vol. 127,2013 , pp. $585-593$. 